University of Nebraska - Lincoln DigitalCommons@University of Nebraska - Lincoln

2002

\title{
High order numerical simulation of sound generated by the Kirchhoff vortex
}

Bernhard Müller

Uppsala University, bernd@tdb.uu.se

Helen C. Yee

NASA Ames Research Center, yee@nas.nasa.gov

Follow this and additional works at: http://digitalcommons.unl.edu/nasapub

Müller, Bernhard and Yee, Helen C., "High order numerical simulation of sound generated by the Kirchhoff vortex" (2002). NASA Publications. 246.

http://digitalcommons.unl.edu/nasapub/246

This Article is brought to you for free and open access by the National Aeronautics and Space Administration at DigitalCommons@University of Nebraska - Lincoln. It has been accepted for inclusion in NASA Publications by an authorized administrator of DigitalCommons@University of Nebraska - Lincoln. 


\title{
Regular article
}

\section{High order numerical simulation of sound generated by the Kirchhoff vortex*}

\author{
Bernhard Müller ${ }^{1}$, H.C. Yee ${ }^{2}$ \\ ${ }^{1}$ Department of Scientific Computing, Information Technology, Uppsala University, P.O. Box 120, S-751 04 Uppsala, Sweden \\ (e-mail: bernd@tdb.uu.se, http://www.tdb.uu.se/ bernd/) \\ 2 NASA Ames Research Center, Moffett Field, CA 94035, USA
}

Received: 31 January $2001 /$ Accepted: 30 September 2001

Communicated by: A. Quarteroni

\begin{abstract}
An improved high order finite difference method for low Mach number computational aeroacoustics (CAA) is described. The improvements involve the conditioning of the Euler equations to minimize numerical cancellation errors, and the use of a stable non-dissipative sixth-order central spatial interior scheme and a third-order boundary scheme. Spurious high frequency oscillations are damped by a thirdorder characteristic-based filter. The objective of this paper is to apply these improvements in the simulation of sound generated by the Kirchhoff vortex.
\end{abstract}

\section{Introduction}

Efficient, low dispersive, stable high order numerical methods are most sought after in the emerging area of computational aeroacoustics (CAA) due to their high accuracy and long time wave propagation requirements $[28,30]$. It has been shown that for appropriate high order methods, the number of grid points per wavelength can be greatly reduced from that of standard second-order spatial schemes [4]. Low dispersive fourth-order or higher linear schemes have been shown to be the methods of choice for linear or weakly nonlinear aeroacoustics in general geometries. Complex and CPU intensive nonlinear schemes such as the fifth or higher-order WENO schemes are generally considered as the method of choice if complex nonlinear aeroacoustic problems are involved. The present study is the first of a series of papers [17, $18]$ in an attempt to combine several of the new developments $[3,5,19,22,23,25,27,31-33]$ in efficient, highly parallelizable high order non-dissipative spatial schemes with characteristic-based filters that exhibit low dispersive long time linear and nonlinear wave propagations [33] for CAA.

\footnotetext{
* RIACS Technical Report 01.02, Jan. 2001, NASA Ames Research Center. Research support for the first author by TFR, the Swedish Research Council for Engineering Sciences, under the project: Numerical Simulation of Vortex Sound; part of the research was conducted as a visiting scientist at RIACS.
}

These papers extend the work of [31-33] for CAA. The goal is to propose a scheme that minimizes numerical cancellation errors, and improves nonlinear stability and accuracy associated with low Mach number CAA without the conventional preconditioning of the discretized compressible governing equations. These papers utilize the aforementioned new developments in an incremental fashion in order to validate the final approach.

The final form of our scheme consists of two levels. From the governing equation level, we condition the Euler equations in two steps. The first step is to split the inviscid flux derivatives into a conservative and a non-conservative portion that satisfies a so-called generalized energy estimate [3, 23]. This involves the symmetrization of the compressible nonlinear Euler equations via a transformation of variables that are functions of the physical entropy [7]. This splitting of the flux derivatives, hereafter, is referred to as the "entropy splitting". The split form of the Euler equations was found to require less numerical dissipation than its un-split counterpart in association with non-dissipative spatial central schemes [32,33]. Owing to the large disparity of acoustic and stagnation quantities in low Mach number aeroacoustics, the second step is to reformulate the split compressible Euler equations with the new unknowns as the small changes of the conservative variables with respect to their large stagnation values [25], hereafter referred to as the "entropy splitting perturbation form". Nonlinearities and the conservative portion of the split flux derivatives are retained. The perturbation form (without entropy splitting) was shown to minimize numerical cancellation errors compared to the original conservation laws [25].

From the numerical scheme level, a sixth-order central interior scheme with a third-order boundary scheme that satisfies a discrete analogue of the integration-by-parts procedure used in the continuous energy estimate (summation-by-parts (SBP) principle) is employed [27]. If the physical boundary conditions (BCs) are implemented correctly and if the split form of the inviscid flux derivatives is used, nonlinear stability of the nonlinear Euler equations $[3,22]$ is obtained. Characteristic and nonreflecting BCs, if needed, are imposed 
at each time step. To suppress the spurious high frequency oscillations associated with central schemes, the exact and a modified version of the characteristic-based filter method of Yee et al. [31] are used. The metric terms in the general coordinate transformation are discretized by the same difference operator as the flow variables leading to freestream preservation (uniform flow conservation) [29] for the conservative portion of the split equations. The time derivative is approximated by a 4-stage low-storage second-order explicit Runge-Kutta method with careful treatment of the intermediate $\mathrm{BC}$ at the different stages of the Runge-Kutta method to minimize the loss of global accuracy of the scheme $[2,4,10]$.

The numerical experiments presented in this paper consider the perturbation form of the Euler equations without entropy splitting, and only a simplified version of the Yee et al. filter is used. Numerical results to gain nonlinear stability (and further minimize the use of numerical dissipation) via the "entropy splitting perturbation form" will be presented in $[17,18]$.

The prediction of vortex sound has been one of the most important goals in CAA, since the noise in turbulent flow is, largely, generated by vortices. Here, we focus on the numerical simulation of a single Kirchhoff vortex with known analytical solution (cf. [16] and Sect. 4). The Kirchhoff vortex is an elliptical patch of constant vorticity rotating with constant angular frequency in irrotational flow. This is a good test case because the high order accuracy of the numerical method for the 2-D Euler equations in general geometries can be checked, and BCs at the surface of a sound generator and at the farfield can be tested.

The outline of the paper is as follows. The perturbation formulation of the Euler equations [25] is reviewed in Sect. 2. The SBP principle of difference operators is reviewed in Sect. 3. The analytical solution for the sound generated by the Kirchhoff vortex is described in Sect. 4. Numerical results are compared with the analytical solution in Sect. 5.

\section{Re-formulation of the Euler equations in a perturbation form}

In low Mach number aeroacoustics, the changes in pressure, density, etc. are much smaller than their reference values. For example, the acoustic pressure $p^{\prime}$ is usually many orders of magnitude lower than the stagnation pressure $p_{0}$. Computing small differences of large numbers on the computer leads to cancellation. The formulation introduced in [25] is used to minimize numerical cancellation error for compressible low Mach number flow. The Euler equations are expressed in terms of the changes of the flow variables with respect to their stagnation values. Since the velocity in stagnant flow is zero and the stagnation conditions are constant, the Euler equations in this perturbation form can be written as

$$
\begin{aligned}
& \frac{\partial \rho^{\prime}}{\partial t}+\nabla \cdot(\rho \boldsymbol{u})^{\prime}=0, \\
& \frac{\partial(\rho \boldsymbol{u})^{\prime}}{\partial t}+\nabla \cdot(\rho \boldsymbol{u})^{\prime} \boldsymbol{u}^{\prime}+\nabla p^{\prime}=0, \\
& \frac{\partial(\rho E)^{\prime}}{\partial t}+\nabla \cdot\left((\rho H)^{\prime} \boldsymbol{u}^{\prime}+(\rho H)_{0} \boldsymbol{u}^{\prime}\right)=0,
\end{aligned}
$$

where

$$
\begin{aligned}
& \rho^{\prime}=\rho-\rho_{0}, \quad(\rho \boldsymbol{u})^{\prime}=\rho \boldsymbol{u}, \quad(\rho E)^{\prime}=\rho E-(\rho E)_{0}, \\
& \boldsymbol{u}^{\prime}=\frac{(\rho \boldsymbol{u})^{\prime}}{\rho_{0}+\rho^{\prime}}, \quad p^{\prime}=(\gamma-1)\left[(\rho E)^{\prime}-\frac{1}{2}(\rho \boldsymbol{u})^{\prime} \cdot \boldsymbol{u}^{\prime}\right], \\
& (\rho H)^{\prime}=(\rho E)^{\prime}+p^{\prime} .
\end{aligned}
$$

Here, $\rho$ denotes the density, $\boldsymbol{u}$ the velocity, $E$ the total energy per unit mass, $H$ the total enthalpy, and $\gamma=1.4$ the ratio of specific heats for air at standard conditions. The "“" and subscript " 0 " denote perturbation and stagnation variables, respectively.

Although this perturbed form is identical to the original conservative laws, discretizing e.g. $\nabla p$ leads to cancellation errors, whereas these errors are minimized when discretizing $\nabla p^{\prime}$. In Cartesian coordinates, the perturbed 2D Euler equations can be expressed as

$$
\frac{\partial \boldsymbol{U}^{\prime}}{\partial t}+\frac{\partial \boldsymbol{F}_{1}^{\prime}}{\partial x}+\frac{\partial \boldsymbol{F}_{2}^{\prime}}{\partial y}=0,
$$

where

$\boldsymbol{U}^{\prime}=\left(\begin{array}{c}\rho^{\prime} \\ (\rho u)^{\prime} \\ (\rho v)^{\prime} \\ (\rho E)^{\prime}\end{array}\right), \boldsymbol{F}_{1}^{\prime}=\left(\begin{array}{l}(\rho u)^{\prime} \\ (\rho u)^{\prime} u^{\prime}+p^{\prime} \\ (\rho v)^{\prime} u^{\prime} \\ (\rho H)^{\prime} u^{\prime}+(\rho H)_{0} u^{\prime}\end{array}\right)$,

$\boldsymbol{F}_{2}^{\prime}=\left(\begin{array}{l}(\rho v)^{\prime} \\ (\rho u)^{\prime} v^{\prime} \\ (\rho v)^{\prime} v^{\prime}+p^{\prime} \\ (\rho H)^{\prime} v^{\prime}+(\rho H)_{0} v^{\prime}\end{array}\right)$

Here, $u^{\prime}=u$ is the $x$-direction velocity and $v^{\prime}=v$ is the $y$ direction velocity.

For the treatment of general geometries, a coordinate transformation $(x(\xi, \eta), y(\xi, \eta))$ is used. The resulting transformed 2D Euler equations are

$$
\frac{\partial \hat{\boldsymbol{U}}^{\prime}}{\partial t}+\frac{\partial \hat{\boldsymbol{F}}_{1}^{\prime}}{\partial \xi}+\frac{\partial \hat{\boldsymbol{F}}_{2}^{\prime}}{\partial \eta}=0,
$$

where

$$
\begin{aligned}
& \hat{\boldsymbol{U}}^{\prime}=J^{-1} \boldsymbol{U}^{\prime}, \\
& \hat{\boldsymbol{F}}_{1}^{\prime}=J^{-1} \xi_{x} \boldsymbol{F}_{1}^{\prime}+J^{-1} \xi_{y} \boldsymbol{F}_{2}^{\prime}, \\
& \hat{\boldsymbol{F}}_{2}^{\prime}=J^{-1} \eta_{x} \boldsymbol{F}_{1}^{\prime}+J^{-1} \eta_{y} \boldsymbol{F}_{2}^{\prime},
\end{aligned}
$$

with the Jacobian determinant of the transformation

$J^{-1}=\frac{\partial x}{\partial \xi} \frac{\partial y}{\partial \eta}-\frac{\partial x}{\partial \eta} \frac{\partial y}{\partial \xi}$, and the metric terms

$J^{-1} \xi_{x}=\frac{\partial y}{\partial \eta}, \quad J^{-1} \xi_{y}=-\frac{\partial x}{\partial \eta}$,

$J^{-1} \eta_{x}=-\frac{\partial y}{\partial \xi}, \quad J^{-1} \eta_{y}=\frac{\partial x}{\partial \xi}$. 


\section{Numerical method}

\subsection{Summation-by-parts $(S B P)$ principle}

For linear partial differential equations, well-posedness of the Cauchy problem or initial-boundary-value problems (IBVPs) can be proved by the energy method [5,12]. The essential mathematical tool in the energy method for continuous problems is integration-by-parts

$\left(u, v_{x}\right)=u(1)^{T} v(1)-u(0)^{T} v(0)-\left(u_{x}, v\right)$.

Here $u$ and $v$ are differentiable d-dimensional real functions on $[0,1]$ and not to be confused with the $u$ and $v$ velocities of the 2D Euler equations. The $(u, v)=\int_{0}^{1} u^{T} v d x$ is the $L_{2}$ scalar product and $\|u\|^{2}=(u, u)$ denotes the $L_{2}$ norm.

As an example, we consider the scalar linear advection equation

$u_{t}+c u_{x}=0, \quad 0 \leq x \leq 1$,

$u(x, 0)=f(x), \quad 0 \leq x \leq 1$,

$u(0, t)=g(t), \quad 0 \leq t$,

where the wave speed $c>0$ is constant. Applying the product rule and (6) to (7), we obtain the equalities

$$
\begin{aligned}
& \frac{d}{d t}\|u(\cdot, t)\|^{2}=2\left(u, u_{t}\right)=-2 c\left(u, u_{x}\right) \\
& =-c\left(u^{2}(1, t)-u^{2}(0, t)\right)=-c u^{2}(1, t)+c g^{2}(t) .
\end{aligned}
$$

Note that $u^{T}=u$ for the scalar problem (7). Integration over a time interval $[0, t]$ shows that the energy $\frac{1}{2}\|u(\cdot, t)\|^{2}$ can be estimated in terms of the initial condition (IC) and BCs. Thus, the problem is well-posed.

Assume the computational domain $[0,1]$ is discretized by $N+1$ grid points $x_{j}=j h, j=0,1, \ldots, N$, with $h=\frac{1}{N}$. Kreiss and Scherer [13], Strand [27] and Carpenter et al. [1] constructed high order difference operators $Q$ for " $d / d x$ " such that the SBP principle holds, i.e.

$(u, Q v)_{h}=u_{N} v_{N}-u_{0} v_{0}-(Q u, v)_{h}$,

where $u, v \in \mathbb{R}^{N+1}$. The discrete scalar product and norm are defined by

$$
(u, v)_{h}=h u^{T} H v, \quad\|u\|_{h}^{2}=(u, u)_{h},
$$

where $H$ is a symmetric positive definite $(N+1) \times(N+1)$ matrix.

We employ a $Q$ operator, which is third-order accurate near the boundary and compatible with the standard sixthorder central difference operator in the interior. It was derived by Strand [27] and is of the form

$$
\left(Q_{x} v\right)_{j}= \begin{cases}\frac{1}{h} \sum_{k=0}^{8} d_{j k} v_{k}, & j=0, \ldots, 5, \\ \left(Q_{x}^{(6)} v\right)_{j}, & j=6, \ldots, N-6, \\ -\frac{1}{h} \sum_{k=0}^{8} d_{N-j, k} v_{N-k}, & j=N-5, \ldots, N,\end{cases}
$$

where $\left(Q_{x}^{(6)} v\right)_{j}=\frac{1}{h}\left(\frac{1}{60} v_{j+3}-\frac{3}{20} v_{j+2}+\frac{3}{4} v_{j+1}-\frac{3}{4} v_{j-1}+\frac{3}{20} v_{j-2}\right.$ $\left.-\frac{1}{60} v_{j-3}\right)$ is the standard sixth-order central difference approximation of the first derivative. The forms of the $6 \times 9$ matrix $D=\left(d_{j k}\right)$ and matrix $H$ can be found in [3,27]. Here $H$ is a diagonal matrix defining the norm of the $Q$ operator. The global order of accuracy for (12) is four. Since (12) is based on a diagonal norm, its application to multi-dimensions is straightforward.

To closely maintain the order of accuracy of the scheme in curvilinear coordinates, the metric terms are discretized by the same difference operators as the flux derivatives in (5). In 3D, the Vinokur and Yee [29] treatment of the corresponding metric terms for freestream preservation is recommended.

In order to satisfy the discrete energy estimate, there are different ways of imposing the physical BCs in conjunction with the $Q$ operator to obtain strict linear stability $[1,20$, 21]. The penalty method called "simultaneous approximation term" (SAT) of Carpenter et al. [1] or the projection method of Olsson $[20,21]$ are two popular approaches. Either approach yields a discrete energy relation similar to the continuous energy relation. Nonlinear stability can be achieved by applying the boundary schemes to the in-going characteristic variables via the entropy splitting form of the inviscid flux derivatives. For simplicity, we have implemented the in-going Riemann invariants without the SAT or the projection operator. We use instead the so-called injection method, i.e. by imposing the $Q$ operator explicitly (cf. Sect. 5) which might destroy strict stability.

\subsection{Time integration}

The application of the spatial discretization of the perturbed Euler equations in transformed coordinates (5) results in a semi-discrete system of nonlinear ODEs

$$
\frac{d \boldsymbol{U}}{d t}=\boldsymbol{R}(\boldsymbol{U})
$$

where $\boldsymbol{U}$ is the vector of the difference approximations $\boldsymbol{U}_{j, k}^{\prime}$ and $\boldsymbol{R}$ is the vector of two-dimensional spatial difference operators operating on $\frac{\partial \hat{\boldsymbol{F}}_{1}^{\prime}}{\partial \xi}$ and $\frac{\partial \hat{\boldsymbol{F}}_{2}^{\prime}}{\partial \eta}$ with each element $\boldsymbol{R}_{j, k}=$ $-J_{j, k}\left[Q_{\xi} \hat{\boldsymbol{F}}_{1}^{\prime}+Q_{\eta} \hat{\boldsymbol{F}}_{2}^{\prime}\right]_{j, k}$.

For efficiency, the ODE system is solved by a multistage method [9]

$$
\begin{aligned}
& \boldsymbol{U}^{(1)}=\boldsymbol{U}^{n}+\frac{\Delta t}{4} \boldsymbol{R}\left(\boldsymbol{U}^{n}\right), \\
& \boldsymbol{U}^{(2)}=\boldsymbol{U}^{n}+\frac{\Delta t}{3} \boldsymbol{R}\left(\boldsymbol{U}^{(1)}\right), \\
& \boldsymbol{U}^{(3)}=\boldsymbol{U}^{n}+\frac{\Delta t}{2} \boldsymbol{R}\left(\boldsymbol{U}^{(2)}\right), \\
& \boldsymbol{U}^{n+1}=\boldsymbol{U}^{n}+\Delta t \boldsymbol{R}\left(\boldsymbol{U}^{(3)}\right) .
\end{aligned}
$$

This time discretization is of $O\left(\Delta t^{2}\right)$ for nonlinear problems and $O\left(\Delta t^{4}\right)$ for linear problems. It has the same stability domain as the classical fourth-order Runge-Kutta method. The CFL conditions for the numerical solution of $\frac{\partial u}{\partial t}+c \frac{\partial u}{\partial x}=0$, $c=$ constant, with periodic IC and BC are (e.g. [15]):

$\sigma \leq 2.828$ for $Q_{x}^{(2)}, \sigma \leq 2.061$ for $Q_{x}^{(4)}, \sigma \leq 1.783$ for $Q_{x}^{(6)}$, where $\sigma=\frac{c \Delta t}{\Delta x}$ is the CFL number and $Q_{x}^{(l)}$ denotes the stan- 
dard central $l$ th-order finite difference method. The difference in the phase errors between the two Runge-Kutta methods will be addressed in a forthcoming paper.

In order to maintain the global order of accuracy of the spatial difference operator and the multistage RungeKutta temporal discretization, one needs to impose the time-dependent physical BC correctly (cf. [4], pp. 202-203). For example, incorrect implementation of a time-dependent Dirichlet BC lacks the 'errors' expected during the different stages of the classical Runge-Kutta method, because the inconsistency ruins the normal cancellation of errors to final global 4th-order spatial accuracy. Instead, the inconsistency leads to $\mathcal{O}(\Delta t)$ and $\mathcal{O}(\Delta x)$ at the boundary and $\mathcal{O}\left(\Delta x^{2}\right)$ globally independent of the high order finite difference operator used. The problem and remedies are discussed in $[2,4,10]$. Here, we choose the remedy, at every intermediate stage of the Runge-Kutta method, of not imposing the time-dependent Dirichlet BCs and employing the $Q$ difference operator at the boundary points. Only after the completion of the full step of the Runge-Kutta method are the time-dependent Dirichlet $\mathrm{BCs}$ prescribed.

\subsection{Characteristic-based filter}

For long time wave propagation of nonlinear systems, even with the absence of shock waves and/or steep gradients, spurious high frequency oscillations are generated by nondissipative central spatial schemes. To suppress these spurious oscillations, a modified version of the Yee et al. [31] high order artificial compressibility method (ACM) filter scheme is used. In the Yee et al. [31,32] scheme, one time step consists of one step with a fourth-order or higher central spatial base scheme for the interior grid points. Often an entropy split form of the inviscid flux derivative is used along with a post processing step, where regions of oscillation are detected using the ACM sensor, and filtered by adding the numerical dissipation portion of a shock capturing scheme at these parts of the solution. The ACM sensor is based on Harten's ACM [6] switch but utilized in a different context. The idea of the scheme is to have the spatially higher nondissipative scheme activated at all times and to add the full strength, efficient and accurate numerical dissipation only at the shock layers, steep gradients and spurious oscillation parts. For the present test problems, we employ a similar procedure but with a simplified filter. The ACM sensor itself, the limiter of the numerical dissipation, and the Roe's averaged states are not used. For low Mach number CAA, the limiter which is designed for capturing discontinuities might not be necessary, unless shear layers are present. For robustness and achieving low dispersive property in general CAA, results from [31-33] indicate that the ACM or wavelet sensor is necessary.

At the completion of a full step of the Runge-Kutta method, the numerical solution $\boldsymbol{U}_{j, k}^{\prime n+1}$ is filtered by a thirdorder difference operator

$\tilde{\boldsymbol{U}}_{j, k}^{\prime n+1}=\boldsymbol{U}_{j, k}^{\prime n+1}-\Delta t J_{j, k}\left[D_{\xi} \boldsymbol{U}^{\prime}+D_{\eta} \boldsymbol{U}^{\prime}\right]_{j, k}^{n+1}$,

$D_{\xi} \boldsymbol{U}_{j, k}^{\prime}=\kappa \delta_{\xi} \boldsymbol{R}_{\xi}\left|\boldsymbol{\Lambda}_{\xi}\right| \delta_{\xi}^{2} \boldsymbol{R}_{\xi}^{-1} \delta_{\xi} \boldsymbol{U}_{j, k}^{\prime}$

with the difference operator $\delta_{\xi} \boldsymbol{U}_{j, k}^{\prime}=\boldsymbol{U}_{j+1 / 2, k}^{\prime}-\boldsymbol{U}_{j-1 / 2, k}^{\prime}$. The Jacobian matrix of the $\xi$-direction flux can be diag- onalized as $\frac{\partial \hat{\boldsymbol{F}}_{1}}{\partial \boldsymbol{U}}=\boldsymbol{R}_{\xi} \boldsymbol{\Lambda}_{\xi} \boldsymbol{R}_{\xi}^{-1}$. The columns of $\boldsymbol{R}_{\xi}$ are the right eigenvectors of $\frac{\partial \hat{\boldsymbol{F}}_{1}}{\partial \boldsymbol{U}}$ and may be found in [29]. The eigenvalues of $\frac{\partial \hat{\boldsymbol{F}}_{1}}{\partial \boldsymbol{U}}$ define the diagonal matrix $\boldsymbol{\Lambda}_{\xi}=$ diag $\left(u_{\xi}-c_{\xi}, u_{\xi}, u_{\xi}, u_{\xi}+c_{\xi}\right)$, where $u_{\xi}=u J^{-1} \xi_{x}+v J^{-1} \xi_{y}$, and $c_{\xi}=c \sqrt{\left(J^{-1} \xi_{x}\right)^{2}+\left(J^{-1} \xi_{y}\right)^{2}}$. The $\boldsymbol{R}_{\xi}$ is evaluated at the arithmetic average. $D_{\eta} \boldsymbol{U}_{j, k}^{\prime}$ is defined analogously. For our numerical experiments, a positive filter coefficient $\kappa$ that is below 0.05 works well for the present test problem. Whether a characteristic filter instead of a scalar filter is absolutely necessary, and the advantage of using the exact form of the Yee et al. ACM sensor or the wavelet sensor [26] will be addressed in a future paper.

\section{Analytical solution for Kirchhoff vortex sound}

The Kirchhoff vortex is an elliptical patch (Fig. 1) with semimajor axis $a$ and semi-minor axis $b$ of constant vorticity $\boldsymbol{\nabla} \times \boldsymbol{u}=(0,0, \omega)^{T}$ rotating with constant angular frequency $\Omega=\frac{a b}{(a+b)^{2}} \omega$ in irrotational flow [11]. The 2D flow field constitutes an exact solution of the 2D incompressible Euler equations [14]. The acoustic pressure generated by the Kirchhoff vortex is governed by the 2D Helmholtz equation, which can be solved analytically using separation of variables [16].

The normal velocity for an almost circular Kirchhoff vortex of radius $R$, i.e. $a=R(1+\epsilon), b=R(1-\epsilon), 0<\epsilon \ll 1$, can be approximated by [16]

$\boldsymbol{u} \cdot \boldsymbol{n} \approx 2 R \epsilon \Omega \sin (2(\theta-\Omega t))$.

Assuming a harmonic time dependence at the angular frequency $2 \Omega$ for the acoustic pressure

$p^{\prime}(r, \theta, t)=\hat{p}(r, \theta) \mathrm{e}^{-\mathrm{i} 2 \Omega t}$,

reduces the wave equation to the Helmholtz equation

$k^{2} \hat{p}+\Delta \hat{p}=0$

with wave number $k=2 \Omega / c_{0}$. Separation of variables yields the solution of the Helmholtz equation

$p^{\prime}(r, \theta, t)=\Re\left(A H_{2}^{(1)}(k r) \mathrm{e}^{\mathrm{i}(2(\theta-\Omega t))}\right)$,

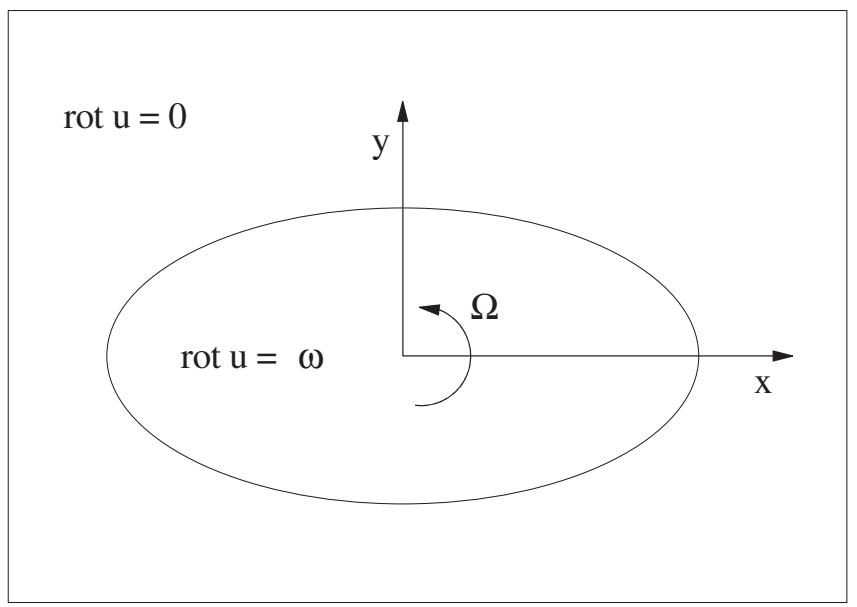

Fig. 1. Kirchhoff vortex 
where $\mathfrak{R}(z)$ denotes the real part of a complex number $z . H_{2}^{(1)}$ is the Hankel function of 2 nd order. The constant

$$
A=\frac{\rho_{0} 4 R \epsilon \Omega^{2}}{k H_{2}^{(1) \prime}(k R)}
$$

is determined by the radial momentum equation

$\rho_{0} \frac{\partial u_{r}}{\partial t}=-\frac{\partial p^{\prime}}{\partial r}$

using the normal velocity (16) of the Kirchhoff vortex. In [16], the farfield approximation of (17) for $k r \gg 1$ is shown to coincide with the farfield approximation derived with Green's function [8], pp. 126-128. An almost circular Kirchhoff vortex generates a similar sound field as an almost circular rotating impermeable ellipse [24].

The exact solution of the 2D linearized Euler equations for sound generated by the Kirchhoff vortex is determined by means of the exact solution of the Helmholtz equation (17). The velocity $\boldsymbol{u}$ and the velocity potential $\varphi$ are obtained by solving

$\boldsymbol{u}=\nabla \varphi, \quad p^{\prime}=-\rho_{0} \frac{\partial \varphi}{\partial t}$.

Integrating over time and using (17), we get

$$
\begin{aligned}
\varphi(r, \theta, t) & =-\frac{1}{\rho_{0}} \int^{t} p^{\prime}(r, \theta, \tau) d \tau \\
& =-\frac{1}{\rho_{0}} \Re\left(A H_{2}^{(1)}(k r) \frac{1}{-2 \Omega i} \mathrm{e}^{\mathrm{i}(2(\theta-\Omega t))}\right) .
\end{aligned}
$$

For isentropic flow, the density perturbation is obtained from $\rho^{\prime}=\frac{1}{c_{0}^{2}} p^{\prime}$

\section{Numerical examples}

In this section we illustrate the accuracy of the high order method by several numerical examples. The time step $\Delta t=$ 0.15 is used for all cases.

\subsection{Rotating Kirchhoff vortex}

We consider a Kirchhoff vortex with radius $R=2 \mathrm{~m}, \epsilon=$ $0.00125, \Omega=82.5 \mathrm{~s}^{-1}$. The stagnation conditions are $\rho_{0}=$ $1.3 \mathrm{~kg} / \mathrm{m}^{3}, c_{0}=330 \mathrm{~m} / \mathrm{s}$. Thus, the Helmholtz number becomes $\mathscr{H}=k R=2 \Omega R / c_{0}=1$.

A polar grid mildly stretched near the Kirchhoff vortex in the radial direction, and uniform in the circumferential direction of $129 \times 24$ is used. We approximate $\frac{\partial}{\partial \xi}$ by (12) and $\frac{\partial}{\partial \eta}$ by the standard sixth-order central difference operator $Q_{\eta}^{(6)}$. The periodic BCs in the circumferential direction are implemented by 6 overlapping grid points. The exact solution of the linearized Euler equations (cf. Sect. 4) is prescribed as the IC. At the circle $r=R$ and at the farfield $r=64.375 \mathrm{~m}$, characteristic BCs are imposed after the completion of a full step of the Runge-Kutta method.Runge-Kutta With the density, velocity and pressure non-dimensionalized with reference quantities $\rho_{0}, c_{0}$ and $\rho_{0} c_{0}^{2}$, respectively, the Riemann invariants can be expressed as $p^{\prime}-u_{n}, p^{\prime}-\rho^{\prime}, u_{t}$ and $p^{\prime}+u_{n}$. Here, $u_{n}$ is the normal velocity and $u_{t}$ is the tangential velocity. The in-going Riemann invariants are prescribed using the exact solution of the linearized Euler equations, while the outgoing Riemann invariants are taken from the numerical solution computed at the boundary. For example, at $r=R$, if $c \geq u_{n}>0$ then $p^{\prime}-u_{n}=\left(p^{\prime}-u_{n}\right)_{\text {computed }}, p^{\prime}-\rho^{\prime}=\left(p^{\prime}-\rho^{\prime}\right)_{\text {exact }}=0$, $u_{t}=\left(u_{t}\right)_{\text {exact }}$ and $p^{\prime}+u_{n}=\left(p^{\prime}+u_{n}\right)_{\text {exact }}$. If $-c<u_{n} \leq 0$, the Riemann invariants for the acoustic waves are unchanged, while those for the entropy and vorticity waves become $p^{\prime}-$ $\rho^{\prime}=\left(p^{\prime}-\rho^{\prime}\right)_{\text {computed }}$ and $u_{t}=\left(u_{t}\right)_{\text {computed }}$.

At $t=30$, the Kirchhoff vortex has rotated 7.5 radians. Figures 2 and 3 show the computed solution along the positive $x$-axis. The solution without the filter compares well with the exact solution of the linearized Euler equations, except near $x=5$, where high frequency oscillations are visible. These spurious oscillations are eliminated by applying

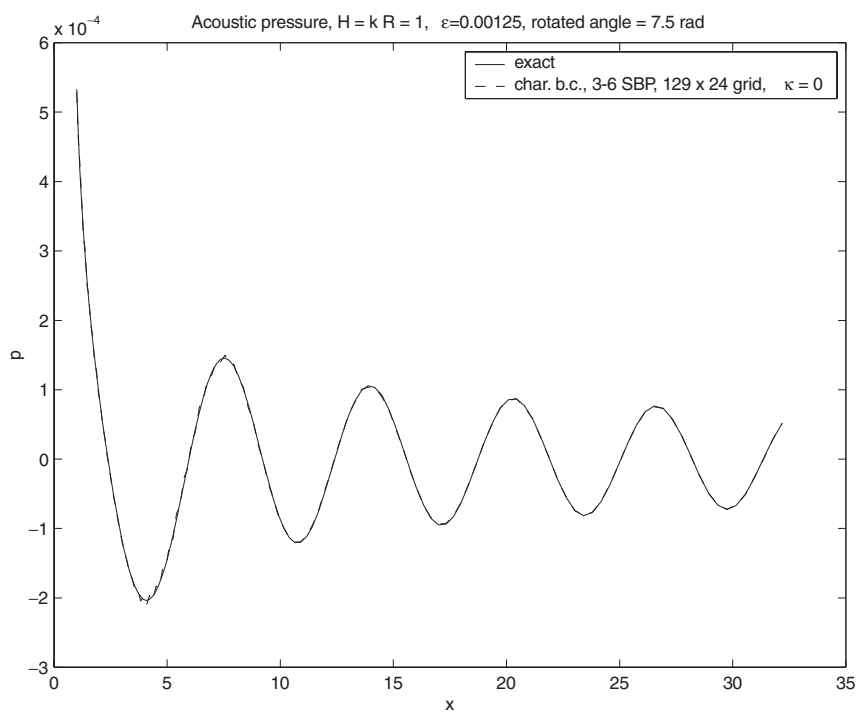

Fig. 2. Comparison of computed and exact acoustic pressure for $\mathscr{H}=1, \epsilon=$ 0.00125 , rotated angle $=7.5 \mathrm{rad}$

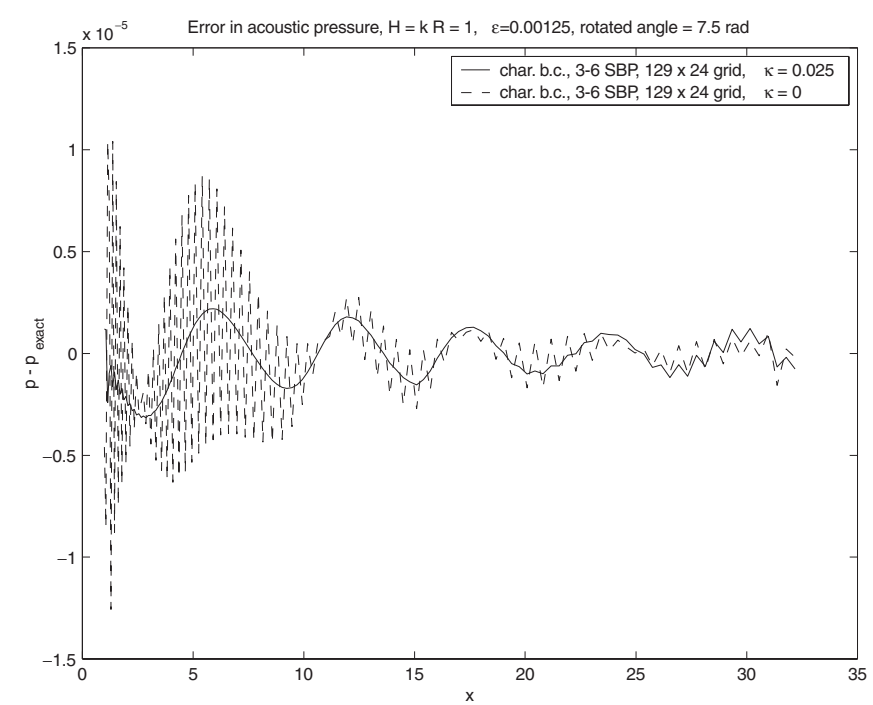

Fig. 3. Influence of filter on error of acoustic pressure for $\mathscr{H}=1, \epsilon=$ 0.00125 , rotated angle $=7.5 \mathrm{rad}$ 
the characteristic-based filter with $\kappa=0.025$. The filtered and the exact solutions agree up to plotting accuracy except in the vicinity of the boundaries (cf. Fig. 3). With larger $\kappa$, e.g. $\kappa=0.05$, the deviations near the boundaries slightly increase. Note that the present simplified filter applies the same amount (although small) of characteristic numerical dissipations everywhere. To achieve low dispersive property, the exact form of the ACM or wavelet sensor [26,31,32] should be employed. Regions of the spurious oscillations are detected precisely by the ACM or wavelet sensor. In addition, these sensors adaptively indicate the appropriate amount of numerical dissipations needed at each grid point. It is noted that while the characteristic length scale was chosen as $L=1 \mathrm{~m}$ in the computation, we use $R=2 \mathrm{~m}$ in the plots, i.e. $x=\frac{x^{*}}{L}$ in the computation, where $x^{*}$ is the $x$-coordinate in $\mathrm{m}$, and $x=\frac{x^{*}}{R}$ in the plots. $p$ in the plots corresponds to the nondimensional acoustic pressure $p^{\prime}$.

\subsection{Kirchhoff vortex started instantaneously}

A Kirchhoff vortex rotating forever was considered in Sect. 5.1. Here, the same problem is computed but with the IC of the Kirchhoff vortex starting instantaneously from stagnation conditions. The ICs are $\rho^{\prime}=u^{\prime}=v^{\prime}=p^{\prime}=0$, except for the circle $r=R$, where the exact solution of the 2D linearized Euler equations for sound generated by the Kirchhoff vortex of Sect. 4 is prescribed at $t=0$. At the circle $r=R$, we use the same characteristic BCs as in Sect. 5.1. At the farfield, we now use nonreflecting BCs. After the completion of a full step of the Runge-Kutta method, the in-going Riemann invariants for stagnation conditions are prescribed. This means that, at the subsonic farfield boundary $p^{\prime}-u_{n}=0$ is imposed. For $u_{n}<0, p^{\prime}-\rho^{\prime}=0$ and $u_{t}=0$ are imposed as well.

The analytical solution of Sect. 4 is not valid for the instantaneously started Kirchhoff vortex. It is only valid for a Kirchhoff vortex which has been rotating forever. However, since no wave is travelling from the farfield towards the Kirchhoff vortex, as long as we have stagnation conditions in the farfield, we can assume that the analytical solution for the Kirchhoff vortex rotating for infinitely long time is valid up to the wavefront of the instantaneously started Kirchhoff vortex. If the wavefront has left the domain without reflection at the farfield, the analytical solutions for the instantaneously started Kirchhoff vortex and for the infinitely long rotating Kirchhoff vortex should agree.

Figures 4-6 show the effect of the characteristic-based filter when the wave front has reached $r \approx 16$ (at $t=30$ ). The numerical solution without filter exhibits spurious oscillations whereas the characteristic-based filter with $\kappa=0.025$ agrees well with the analytical solution between $r=1$ and $r \approx 14$. Their difference in accuracy is more apparent from the acoustic pressure shown in Fig. 6 . At $x \approx 16$, we see that in general the wavefront cannot match the infinitely long rotating Kirchhoff vortex solution, because the instantaneously started Kirchhoff vortex has zero acoustic pressure downstream of the wavefront. The discrepancies between the numerical solution for the instantaneously started Kirchhoff vortex and the analytical solution for the infinitely long rotating Kirchhoff vortex, therefore, have physical reasons.

Next, we consider the same instantaneously started Kirchhoff vortex as before, but compute for a longer time. At

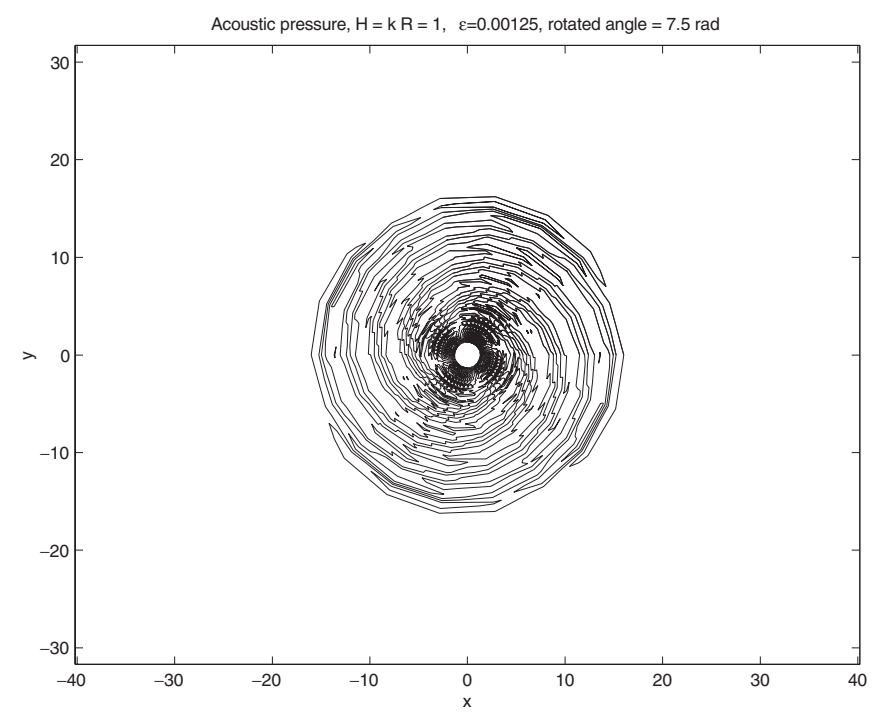

Fig. 4. Acoustic pressure contours without filter for instantaneously started Kirchhoff vortex with $\mathscr{H}=1, \epsilon=0.00125$, rotated angle $=7.5 \mathrm{rad}$

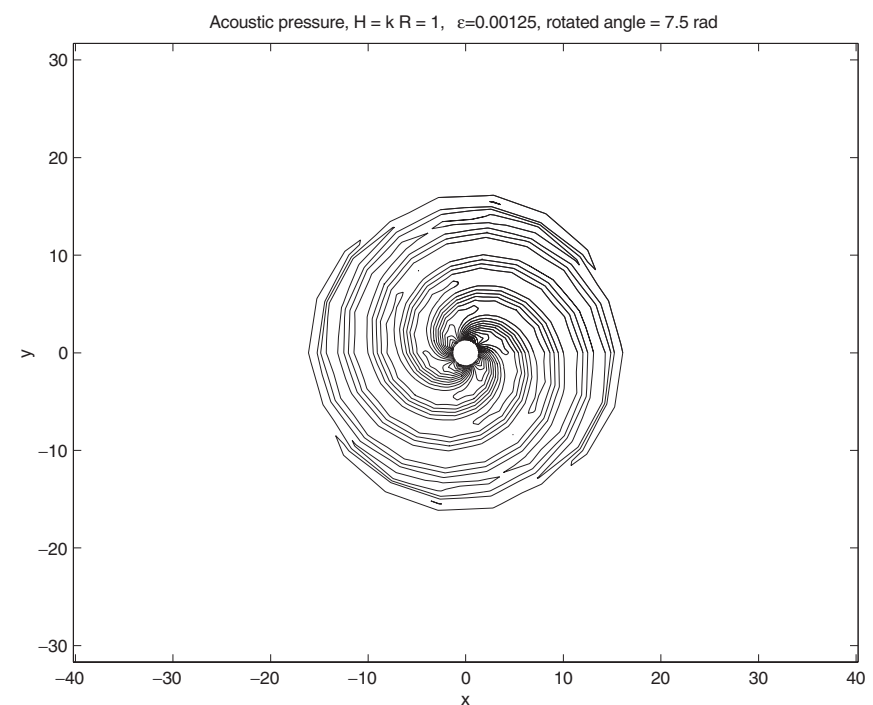

Fig. 5. Acoustic pressure contours with filter for instantaneously started Kirchhoff vortex with $\mathscr{H}=1, \epsilon=0.00125$, rotated angle $=7.5 \mathrm{rad}$

$t=75$, the Kirchhoff vortex has rotated 18.75 radians. Now, the wavefront is at $r \approx 38.5$. If the wavefront were reflected at the farfield $r=32.1875$, we should see it at $r \approx 25.875$. The computed acoustic pressure along the positive $x$-axis shown in Fig. 7 is in excellent agreement with the analytical solution, if the characteristic-based filter with $\kappa=0.025$ is used. Without the filter, spurious oscillations can be clearly seen. It is interesting to note that even without the filter, the wavefront generated at $t=0$ by the Kirchhoff vortex has passed through the farfield without visible reflection. The quadrupole structure of the acoustic pressure is correctly recovered by the high order $Q$ operator with the characteristic-based filter $\kappa=0.025$.

A grid refinement study with a slightly modified filter operator $D_{\xi} \boldsymbol{U}_{j, k}^{\prime}=0.01 \delta_{\xi}^{2} \boldsymbol{R}_{\xi}\left|\boldsymbol{\Lambda}_{\xi}\right| \boldsymbol{R}_{\xi}^{-1} \delta_{\xi}^{2} \boldsymbol{U}_{j, k}^{\prime}$ and similar $D_{\eta}$ in (15) yields the expected convergence rate of 4 , when using $65 \times 15$ and $129 \times 24$ grids. However, when refining from $129 \times 24$ to $257 \times 42$ grids, the convergence rate drops to 


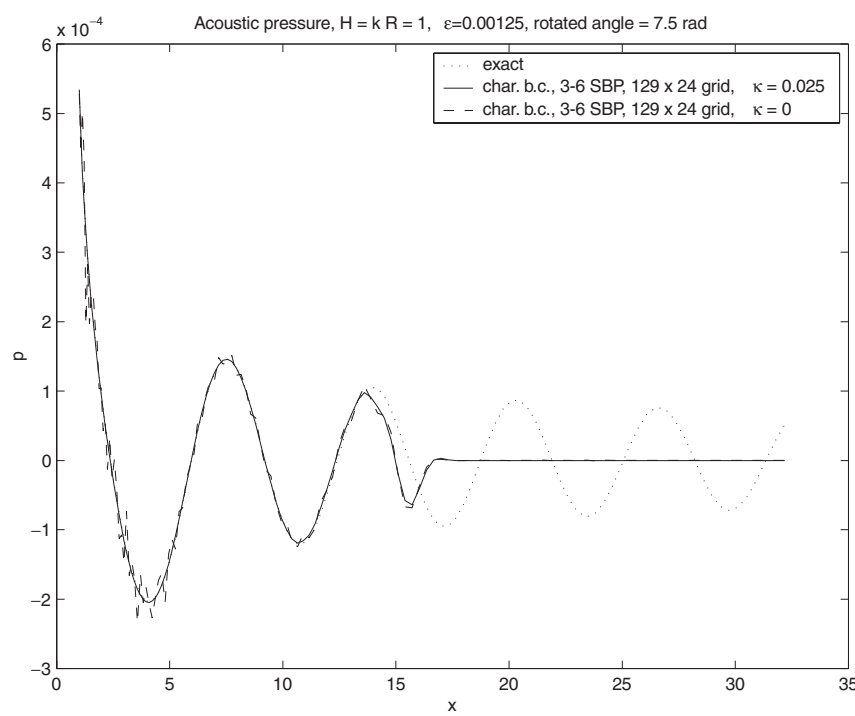

Fig. 6. Comparison of acoustic pressure for instantaneously started Kirchhoff vortex with $\mathscr{H}=1, \epsilon=0.00125$, rotated angle $=7.5 \mathrm{rad}$

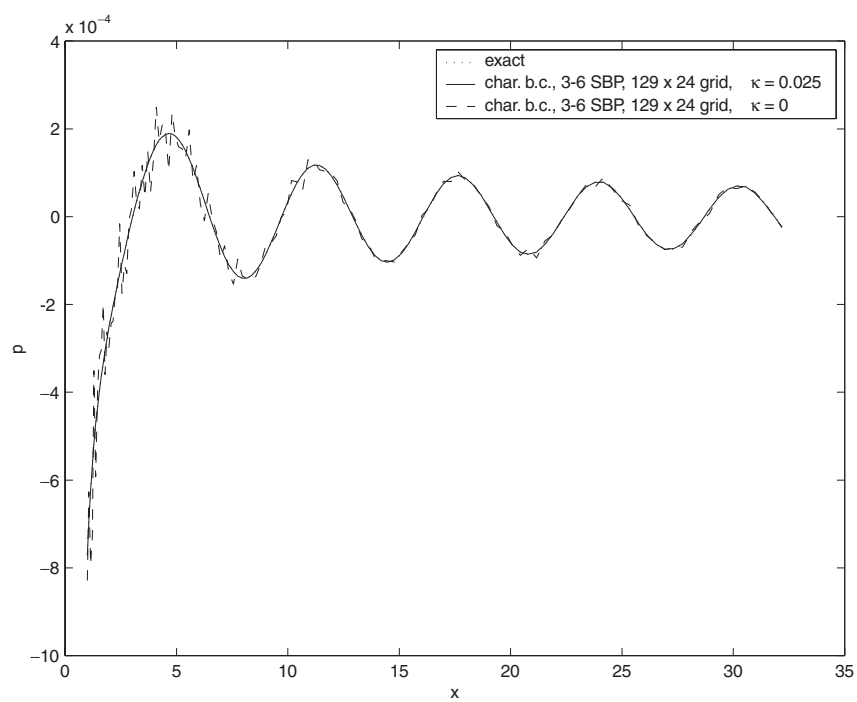

Fig. 7. Comparison of acoustic pressure for instantaneously started Kirchhoff vortex with $\mathscr{H}=1, \epsilon=0.00125$, rotated angle $=18.75 \mathrm{rad}$

about 3 for $p, \rho$, and $\rho E$ and to about 1.8 and 1.6 for $u$ and $v$, respectively. Errors in the tangential velocity in the vicinity of the Kirchhoff vortex are due to spurious oscillations probably caused by the boundary treatment. Doubling the filter parameter for the $x$ - and $y$-momentum equations eliminates the oscillations but reduces the accuracy. Nevertheless, the present difference operator $Q$, which is sixth-order accurate in the interior and third-order near the boundary, is far superior to the conventional second-order difference approximation with one-sided first-order difference methods at the boundaries. For nearly the same CPU time on a $129 \times 24$ grid, the $l_{2}$-errors of the flow variables are about 10 times lower with the high order method. Figure 8 shows the comparison of the $x$-velocity component on the $x$-axis at $t=75$. The dispersion error of the low order method is clearly visible. Even on a $257 \times 42$ grid, the low order results are inferior to the high order ones on the $129 \times 24$ grid. Since the time step is halved

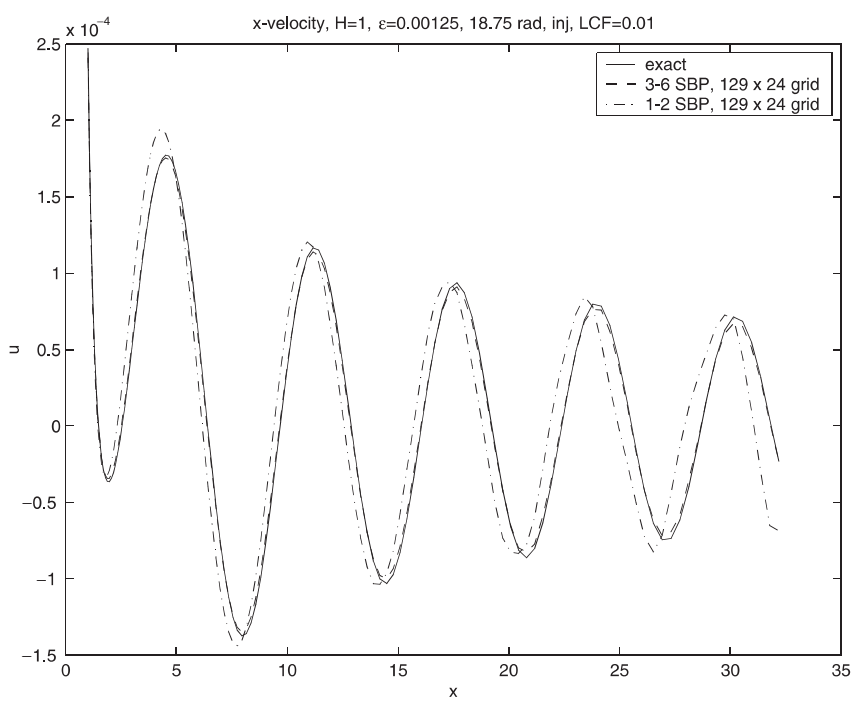

Fig. 8. Comparison of $x$-velocity component using high and low order difference methods for instantaneously started Kirchhoff vortex with $\mathscr{H}=1$, $\epsilon=0.00125$, rotated angle $=18.75 \mathrm{rad}$

with grid refinement, the high order method is more than 8 times more efficient than the low order method in this case.

\section{Conclusions}

A spatially sixth-order interior scheme with a third-order boundary scheme [27] has been used to solve the 2D Euler equations in perturbation form [25] for the sound generated by the Kirchhoff vortex [16]. Spurious oscillations associated with non-dissipative central schemes are eliminated by a characteristic-based filter similar to [31]. The metric terms in the general coordinate transformation are discretized by the same difference operator. Careful treatment of the intermediate $\mathrm{BC}$ at the different stages of the Runge-Kutta method for time-dependent physical BC to minimize loss of global accuracy is employed. Very accurate results are obtained with a fairly coarse grid.

In order to gain nonlinear stability for the nonlinear Euler equations in the hope of further minimizing the use of numerical dissipation, future work includes the use of the entropy splitting form of the Euler equations before the application of the perturbation form, and the use of SAT or the projection method of imposing the physical BC with the $Q$ operator to satisfy the discrete energy estimate. The ACM and wavelet filter sensors $[26,31,32]$ and other possible problemindependent coefficients of the characteristic-based filter will be sought.

\section{References}

1. Carpenter, M.H., Gottlieb, D., Abarbanel, S.: Time-Stable Boundary Conditions for Finite-Difference Schemes Solving Hyperbolic Systems: Methodology and Application to High-Order Compact Schemes. J. Comput. Phys. 111, 220-236 (1994)

2. Carpenter, M.H., Gottlieb, D., Abarbanel, S., Don, W.-S.: The Theoretical Accuracy of Runge-Kutta Time Discretizations for the Initial Boundary Value Problem: A Study of the Boundary Error. SIAM J. Sci. Comput. 16(6), 1241-1252 (1995) 
3. Gerritsen, M., Olsson, P.: Designing an Efficient Solution Strategy for Fluid Flows. J. Comput. Phys. 129, 245-262 (1996)

4. Fornberg, B.: A Practical Guide to Pseudospectral Methods. First Paperback Edition. Cambridge: Cambridge University Press 1998

5. Gustafsson, B., Kreiss, H.-O., Oliger, J.: Time Dependent Problems and Difference Methods. New York: John Wiley \& Sons 1995

6. Harten, A.: The Artificial Compression Method for Computation of Shocks and Contact Discontinuities: III. Self-Adjusting Hybrid Schemes, Math. Comp. 32(142), 363-389 (1978)

7. Harten, A.: On the Symmetric Form of Systems of Conservation Laws with Entropy. J. Comput. Phys. 49, 151-164 (1983)

8. Howe, M.S.: Acoustics of Fluid-Structure Interactions. Cambridge: Cambridge University Press 1998

9. Jameson, A., Baker, T.J.: Solution of the Euler Equations for Complex Configurations. AIAA Paper, 83-1929, 1983

10. Johansson, M.: Loss of High Order Spatial Accuracy due to Boundary Error Caused by Runge-Kutta Time Integration. Technical Report 2000-013, Department of Information Technology, Uppsala University, May 2000. http://www.it.uu.se/research/reports/2000-013/

11. Kirchhoff, G.: Vorlesungen über mathematische Physik. Mechanik. 2nd edn. Leipzig 1877

12. Kreiss, H.-O., Lorenz, J.: Initial-Boundary Value Problems and the Navier-Stokes Equation. New York: Academic Press 1989

13. Kreiss, H.-O., Scherer, G.: Finite Element and Finite Difference Methods for Hyperbolic Partial Differential Equations. In: Mathematical Aspects of Finite Elements in Partial Differential Equations. New York: Academic Press 1974

14. Lamb, H.: Hydrodynamics. 7th edn. Cambridge: Cambridge University Press 1975

15. Lele, S.K.: Compact Finite Difference Schemes with Spectral-like Resolution. J. Comput. Phys. 103, 16-42 (1992)

16. Müller, B.: On Sound Generation by the Kirchhoff Vortex. Report No. 209/1998, Department of Scientific Computing, Uppsala University, Oct. 1998.

http://www. tdb.uu.se/archive/reports/report-209-1998.ps.gz

17. Müller, B., Yee, H.C.: Entropy Splitting for High Order Numerical Simulation of Vortex Sound at Low Mach Numbers. J. Sci. Comput., to appear 2002

18. Müller, B.: High Order Difference Method for Low Mach Number Aeroacoustics. ECCOMAS CFD Conference 2001, Swansea, Wales, UK, Sept. 4-7, 2001
19. Nordström, J., Carpenter, M.H.: Boundary and Interface Conditions for High-Order Finite-Difference Methods Applied to the Euler and Navier-Stokes Equations. J. Comput. Phys. 148, 621-645 (1999)

20. Olsson, P.: Summation by Parts, Projections and Stability. I. Math. Comp. 64(211), 1035-1065 (1995)

21. Olsson, P.: Summation by Parts, Projections and Stability. II. Math. Comp. 64(212), 1473-1493 (1995)

22. Olsson, P.: Summation by Parts, Projections and Stability. III. RIACS Technical Report 95.06, 1995

23. Olsson, P., Oliger, J.: Energy and Maximum Norm Estimates for Nonlinear Conservation Laws. RIACS Technical Report 94.01, 1994

24. Polifke, W., Paschereit, C.O.: Sound Emissions of Rotor Induced Deformations of Generator Casings. Report, ABB Corporate Research, Baden, Switzerland, 1997

25. Sesterhenn, J., Müller, B., Thomann, H.: On the Cancellation Problem in Calculating Compressible Low Mach Number Flows. J. Comput. Phys. 151, 597-615 (1999)

26. Sjögreen, B., Yee, H.C.: Multiresolution Wavelet Based Adaptive Numerical Dissipation Control for Shock-Turbulence Computations. RIACS Technical Report 01.01, October 2000, NASA Ames Research Center

27. Strand, B.: Summation by Parts for Finite Difference Approximations for $d / d x$. J. Comput. Phys. 110, 47-67 (1994)

28. Tam, C.K.W.: Computational Aeroacoustics: Issues and Methods. AIAA J. 33(10), 1788-1796 (1995)

29. Vinokur, M., Yee, H.C.: Extension of Efficient Low Dissipation High Order Schemes for 3-D Curvilinear Moving Grids. NASA TM 209598, 2000, and Proceedings of Computing the Future III: Frontiers of CFD-2000, Half Moon Bay, CA, June 26-28, 2000

30. Wells, V.L., Renaut, R.A.: Computing Aerodynamically Generated Noise. Annu. Rev. Fluid Mechanics 29, 161-199 (1997)

31. Yee, H.C., Sandham, N.D., Djomehri, M.J.: Low-Dissipative HighOrder Shock-Capturing Methods Using Characteristic-Based Filters. J. Comput. Phys. 150, 199-238 (1999)

32. Yee, H.C., Vinokur, M., Djomehri, M.J.: Entropy Splitting and Numerical Dissipation. J. Comput. Phys. 162, 33-81 (2000)

33. Yee, H.C., Sjögreen, B., Sandham, N.D., Hadjadj, A.: Progress in the Development of a Class of Efficient Low Dissipative High Order Shock-Capturing Methods. RIACS Technical Report 00.11, June, 2000, NASA Ames Research Center; Proceedings of the CFD for the 21st Century, July 15-17, 2000, Kyoto, Japan 